\title{
The burden of maternal morbidity and mortality attributable to hypertensive disorders in pregnancy: a prospective cohort study from Uganda
}

Annettee Nakimuli ${ }^{1}$, Sarah Nakubulwa', Othman Kakaire', Michael Odongo Osinde², Scovia Nalugo Mbalinda ${ }^{3}$, Nelson Kakande ${ }^{4}$, Rose Chalo Nabirye ${ }^{3}$ and Dan Kabonge Kaye ${ }^{1 *}$

\begin{abstract}
Background: Hypertensive disorders of pregnancy are a major cause of morbidity and mortality. The objective was to estimate the disease burden attributable to hypertensive disorders of pregnancy in two referral hospitals in Uganda.

Methods: Through a prospective cohort study conducted in Jinja and Mulago hospitals in Uganda from March 1, 2013 and February 28, 2014, hypertension-related cases were analyzed. Maternal near miss cases were defined according to the WHO criteria. Maternal deaths were also analyzed. The maternal near miss incidence ratio, the case-specific severe maternal outcome ratio, the case-specific maternal mortality ratio and the case-fatality ratio were computed.

Results: Of 403 women with hypertensive disorders of pregnancy, 218 (54.1\%) had severe preeclampsia, 172 (42.7 \%) had eclampsia, and 13 had chronic hypertension or Hemolysis, elevated liver enzymes or low platelets (HELLP) syndrome. The case-specific maternal near miss incidence ratios was 8.60 per 1,000 live births for all hypertensive disorders, 3.06 per 1,000 live births for severe preeclampsia and 5.11 per 1,000 live births for eclampsia. The casespecific severe maternal outcome ratio was 9.37 per 1,000 live births for all hypertensive disorders, and was 3.25 per 1,000 live births for severe preeclampsia and 5.61 per 1,000 live births for eclampsia. The case-specific maternal mortality ratio was 780 per 100,000 live births for all hypertensive disorders, and was 1940 per 100,000 live births for severe preeclampsia and 501 per 100,000 live births for eclampsia. The case-fatality ratio was $5.1 \%$ overall (for all hypertensive disorders), but was 8 times higher for eclampsia compared to severe preeclampsia. Cyanosis, abnormal respiration, oliguria, circulatory collapse, coagulopathy, thrombocytopenia, and elevated serum lactate were significantly associated with severe maternal outcomes.

Conclusion: There is high morbidity attributable to hypertensive disorders in pregnancy. Since some of the complications associated with morbidity can be recognized early, it is possible to prevent severe morbidity through early intervention with delivery, antihypertensive therapy and prophylactic magnesium sulphate treatment. The findings highlight the feasibility of implementing a facility-based surveillance system for severe maternal morbidity due to hypertensive disorders.
\end{abstract}

\footnotetext{
* Correspondence: dankkaye@yahoo.com

${ }^{1}$ Department of Obstetrics and Gynecology, School of Medicine, College of

Health Sciences, Makerere University, P.O. Box 7072, Kampala, Uganda

Full list of author information is available at the end of the article
} 


\section{Background}

Hypertensive disorders in pregnancy are a major cause of morbidity and mortality among women and their offspring [1-4]. The burden of disease is a measure that assesses and compares the relative impact of different diseases or disorders on populations by quantifying health loss due to disease (or disorders) that remains after prevention, treatment, or rehabilitation efforts of the health system and society. For hypertensive disorders in pregnancy, the burden is not well documented in many countries worldwide, and few countries have national data on their incidence [5]. From systematic reviews, the crude incidence of eclampsia ranged from 0.1 to $8 \%$ [5-7]. The case fatality ratio of eclampsia ranges from 0 to $1.8 \%$ in high-income countries to $17.7 \%$ in middle-income countries such as India, which reflects the gap in the quality of maternal health care [8-10]. Indeed, while no maternal death due to eclampsia occurred in a one-year period in Sweden [9], one hospital in India reported 11 eclampsia-related deaths [10].

There are several explanations for the high morbidity burden of severe preeclampsia and other hypertensive disorders in pregnancy. Preeclampsia is a disorder characterized by abnormal placentation with subsequent maternal inflammatory and vascular response. It manifests as a systemic inflammatory disease that may lead to multiple maternal organ damage in liver, kidneys, lungs, and central nervous system [11]. The placenta-related complications of the disorders include placental insufficiency, placental abruption, intrauterine growth restriction, preterm birth and intrauterine fetal death [11]. Related systemic complications of the disorders include thrombocytopenia, disseminated intravascular coagulation, acute pulmonary edema, cerebro-vascular disorders, and chronic hypertension, whose risk, in comparison to women without hypertension, increases by 3 to 25 times [12-14]. Local data from Uganda on the magnitude of the morbidity burden of hypertensive diseases was scanty. In particular, data where the WHO criteria of organ-system dysfunction [15] have been used to indicate severity of obstetric complications is not available. Yet these criteria have been validated. The objective was to assess the burden of severe maternal outcomes attributable to hypertensive disorders in pregnancy.

\section{Methods}

\section{Study setting and design}

This was a prospective cohort study of women admitted with pregnancy complications between March 1, 2013 and February 28, 2014. The study was conducted at Mulago and Jinja Hospitals. Mulago is Uganda's national referral hospital and the teaching hospital for Makerere University. The hospital has more than 1500 beds, of which around 400 are maternity beds, and conducts over
30,000 deliveries per year. It has an 8-bed high dependency unit (HDU), a 6-bed severe preeclampsia unit and a 6-bed intensive care unit (ICU), where patients with severe forms of the hypertensive disorders are managed. Jinja is a large regional referral hospital that serves six district hospitals in Central and Eastern Uganda, with about 1000 beds of which around 200 are maternity beds and conducts more than 10,000 deliveries per year, but lacks intensive care facilities. All eligible patients with severe preeclampsia routinely receive magnesium sulfate for eclamptic seizure prophylaxis, in addition to parenteral antihypertensive therapy. Most patients referred with severe preeclampsia receive magnesium sulfate prior to transfer to the regional referral hospitals, as magnesium sulphate is one of the essential drugs available at district hospitals and peripheral health centres that provide basic emergency obstetric care. At the referral units, routine care for admitted patients with severe preeclampsia includes anticonvulsant and antihypertensive therapy, as well as monitoring of maternal well being (6- hourly pulse, blood pressure, urine output and general condition assessment), maternal investigations (for hepatic, hematological and renal dysfunction); and monitoring of fetal well being (uterine and umbilical artery Doppler in addition to 6-hourly fetal heart assessment).

\section{Data collection}

The inclusion criteria was a diagnosis of severe preeclampsia (blood pressure of 160/100 mmHG or above, with albuminuria, with or without laboratory evidence of hematological dysfunction). Women with the above diagnosis who consented to participate were recruited in the study, and were followed up to six months postpartum or death. Using an interviewer-administered questionnaire and through review of medical records, data was collected on socio-demographic characteristics, obstetric history, current pregnancy complications and pregnancy outcomes up to hospital discharge. All participants were then followed up to 12 weeks postpartum. Hypertensive disorders of pregnancy were classified into gestational hypertension, chronic hypertension, mild and severe preeclampsia and eclampsia according to the ACOG guidelines [1]. Data was collected on hematological, hepatic and renal function, in addition to biochemical investigations serum uric acid, lactate and electrolytes. The diagnosis of chronic hypertension was made from presence of hypertension in the first-trimester or a history of co-existing hypertension preceding the pregnancy. Patients with features of hepatic dysfunction, haemolysis or thrombocytopenia were classified as Hemolysis, Elevated liver enzymes, Low platelets (HELLP) syndrome if their presentation fitted the diagnostic criteria for HELLP syndrome. 
Maternal near misses were classified using the WHO criteria for definition of maternal near miss [15]. Shock was defined as a reduction of $40 \mathrm{~mm} \mathrm{Hg}$ of systolic Blood Pressure from baseline despite adequate fluid resuscitation, along with presence of perfusion abnormalities (such as oliguria and lactic acidosis) or presence of acute altered mental status. Acute respiratory distress syndrome (ARDS) was diagnosed according to the American-European Consensus Conference on ARDS [16]. Maternal mortality was considered as death occurring during pregnancy or within six weeks of delivery.

\section{Data analysis}

Descriptive analysis was done whereby categorical variables are presented as frequencies and percentages while numerical variables are presented as means or medians (with standard deviations or inter-quartile ranges respectively). The following indicators for morbidity due hypertensive disorders were computed to highlight the burden due to the hypertensive disorders:

i) The case-specific maternal near miss incidence ratios due to hypertensive disorders was derived as the ratio of near miss per 1,000 live births respectively;

ii) The case-specific severe maternal outcome ratio due to hypertensive disorders as ratio of maternal death plus near misses per 1,000 live births;

iii) The case-fatality ratio due to hypertensive disorders determined as the proportion of deaths out of the total number of patients presenting with hypertensive disorders, expressed as a percentage;

iv) The case-specific severe maternal mortality index due to hypertensive disorders, derived as maternal deaths divided by (total deaths plus maternal near misses) expressed as a percentage;

v) The case-specific maternal mortality ratio due to hypertensive disorders expressed as all maternal deaths per 100,000 live births

Maternal complications and procedures used for patient management were compared among women who presented with the different clinical types of hypertensive disorders. The differences across the groups were assessed with Chi-square test and a p-value for trend reported.

\section{Ethical considerations}

This research was part of a post-doctoral research project of the last author (DKK) entitled:

"Evaluation and surveillance of the impact of maternal and neonatal near-miss morbidity on the health of mothers and infants in Jinja and Mulago hospitals". Ethical approval to conduct the study was obtained from the Ethics and research committees of Mulago hospital
(REC 310-2012), the School of Medicine, Makerere University College of Health Sciences (REC 2012-172) and from Uganda National Council for Science and Technology. Permission to conduct the study was obtained from the department of Obstetrics and Gynaecology, Makerere University, and from Mulago National Referral Hospital and Jinja Hospital.

Participants gave written informed consent to be enrolled in the study and for their data to be included in the study. The languages used in the informed consent were English and the local languages (Luganda and Lusoga for Mulago and Jinja hospitals respectively). Participants included minors (aged 14-17years), as Uganda national guidelines for human subject research allow research on mature and emancipated minors in certain situations (such as in pregnancy), with prior approval of an institutional review board.

For those with very severe morbidity, consent was obtained retrospectively when they recovered, or consent was obtained from the next of kin to involve the patients' in the study and/or to include the patients' information in our data. Participants and their next of kin received assurances that participation was voluntary, and that participants were free to stop participation at any time without their decision affecting the care they were entitled to. All those with complications, and their newborns, were provided free medical care or, where necessary, were offered additional counseling or referred to get other support services not available at the two health facilities. Permission was obtained from the management of the two referral hospital (and from the study participants) to review the participants' records.

\section{Results}

Of the 3100 women with severe obstetric complications, 403 (13.0 \%) had hypertensive disorders. Table 1 shows the characteristics of women with hypertensive disorders. The different groups of hypertensive disorders differed significantly across age group, parity category, and mode of delivery, but did not differ according to timing of the disorder (before or after hospital admission) or on referral status. The prevalence of caesarean section was $73.0 \%$ overall, but was $13 \%$ higher for severe preeclampsia compared to eclampsia. However, eclampsia cases had twice as high incidence of severe maternal outcomes (maternal death and near miss combined) or maternal death (compared to severe preeclampsia). Table 2 shows the maternal morbidity indicators associated with hypertensive disorders. HELLP syndrome had the highest case-specific severe maternal mortality index (maternal deaths divided by total maternal deaths plus maternal near misses) as a percentage.

Table 3 shows the incidence of the different types of hypertensive disorders. Of these, 218 (54.1\%) were severe preeclampsia, while 172 (42.7\%) had eclampsia, and 
Table 1 Characteristics of women with hypertensive disorders of pregnancy

\begin{tabular}{|c|c|c|c|c|c|c|}
\hline \multirow[t]{2}{*}{ Characteristics } & All participants & $\begin{array}{l}\text { Severe } \\
\text { preeclampsia }\end{array}$ & Eclampsia & $\begin{array}{l}\text { Chronic } \\
\text { hypertension }\end{array}$ & HELLP syndrome & \multirow[t]{2}{*}{ p-value } \\
\hline & $n=403 n(\%)$ & $n=218 n(\%)$ & $n=172 \mathrm{n}(\%)$ & $n=4 \mathrm{n}(\%)$ & $n=9 n(\%)$ & \\
\hline Age category (years) & & & & & & $<0.001$ \\
\hline Age $<19$ & $45(11.2)$ & $7(3.2)$ & $37(21.5)$ & $0(0.0)$ & $1(21.2)$ & \\
\hline $19-35$ & $168(41.7)$ & 89 (40.8) & $74(43.0)$ & $1(25.0)$ & $4(44.4)$ & \\
\hline$<36$ & $190(47.1)$ & $122(56.0)$ & $61(35.5)$ & $3(75.0)$ & $4(44.4)$ & \\
\hline Parity & & & & & & 0.017 \\
\hline Primipara & $167(41.4)$ & $83(38.1)$ & $78(45.3)$ & $1(25.0)$ & $5(55.5)$ & \\
\hline Para2-4 & $176(43.7)$ & 99 (45.4) & $74(43.0)$ & $0(0.0)$ & $3(33.3)$ & \\
\hline Parity 5 or higher & $60(14.9)$ & $36(16.5)$ & $20(11.7)$ & $3(75.0)$ & $1(11.2)$ & \\
\hline Referral status & & & & & & 0.493 \\
\hline Yes & $265(65.8)$ & $141(64.7)$ & $113(65.7)$ & $3(75.0)$ & $8(88.9)$ & \\
\hline No & $138(34.2)$ & 77 (35.3) & $59(34.3)$ & $1(25.0)$ & $1(11.1)$ & \\
\hline$\Omega$ Timing of complications & & & & & & 0.907 \\
\hline Occurred prior to admission & $183(45.4)$ & $101(46.3)$ & $76(44.2)$ & $2(50.0)$ & $4(44.4)$ & \\
\hline $\begin{array}{l}\text { Occurred prior to admission and new } \\
\text { complications occurred after admission }\end{array}$ & $137(34.0)$ & $71(32.6)$ & $60(34.9)$ & $2(50.0)$ & $4(44.4)$ & \\
\hline Occurred after admission & $83(20.6)$ & $46(21.1)$ & $36(20.9)$ & $0(0.0)$ & $1(22.2)$ & \\
\hline Mode of delivery & & & & & & 0.001 \\
\hline Vaginal & $90(22.3)$ & $33(15.1)$ & $51(29.6)$ & $1(25.0)$ & $5(55.5)$ & \\
\hline Caesarean delivery & $294(73.0)$ & $175(80.3)$ & $113(65.7)$ & $3(75.0)$ & $4(44.5)$ & \\
\hline Vacuum extraction & $19(4.7)$ & $14(14.6)$ & $5(4.7)$ & $0(0.0)$ & $0(0.0)$ & \\
\hline Severe maternal outcomes & & & & & & 0.001 \\
\hline Yes & $162(40.2)$ & $84(38.5)$ & $144(83.7)$ & $4(100.0)$ & $9(100.0)$ & \\
\hline No & $241(59.8)$ & $134(61.5)$ & $28(16.3)$ & $0(0.0)$ & $0(0.0)$ & \\
\hline Maternal death & & & & & & 0.009 \\
\hline Yes & $20(5.0)$ & $7(3.2)$ & $13(7.6)$ & $0(0.0)$ & $2(22.2)$ & \\
\hline No & $38395.0)$ & $211(96.8)$ & $157(92.4)$ & $4(100.0)$ & $7(77.8)$ & \\
\hline
\end{tabular}

$\Omega$ Timing of complication in relation to admission

13 included HELLP syndrome and chronic hypertension. Of the 403, 265 (65.8\%) were referrals: 141 (64.7\%) of the 218 with severe preeclampsia were referrals, while 113 (65.7\%) of the 172 women with eclampsia were referrals.

Table 4 shows the characteristics of the participants assessed according to the diagnostic criteria for definition of severe maternal outcomes (maternal near miss cases and maternal deaths. Cyanosis, abnormal respiration, oliguria, loss of consciousness, circulatory collapse or shock, coagulopathy, presence of thrombocytopenia, elevated serum lactate, the need for dialysis, and admission to the high dependency unit were significantly associated with severe maternal outcomes for eclampsia compared to severe preeclampsia.

\section{Discussion}

Our study shows that hypertensive disorders of pregnancy constitute a high burden of morbidity and mortality as indicated by the morbidity and mortality indices. As noted in literature [17-20], the disorders include chronic (pre-existing) hypertension, gestational (transient) hypertension, preeclampsia/eclampsia, preeclampsia superimposed on chronic hypertension and eclampsia. Both gestational hypertension and preeclampsia/eclampsia may be associated in later life with hypertension and/or cardiovascular disease [17-20].

The burden of hypertensive disorders of pregnancy seems higher than that from the published literature from high income countries [1-4]. While the case fatality rate for eclampsia ranged from 0 to $1.8 \%$ in highincome countries [8], it was $17.7 \%$ in India [10] and was 2.3 in 2014 in a study from Uganda [21]. Such a wide range is due to both differences in incidence and quality of obstetric care for hypertensive disease in pregnancy. For our study, the high burden is associated with delayed recognition of the hypertensive disorders, delay in 
Table 2 Maternal morbidity and mortality indicators associated with hypertensive disorders of pregnancy

\begin{tabular}{|c|c|c|}
\hline Indicator & Overall ratio & Case-specific ratio \\
\hline \multirow{4}{*}{$\begin{array}{l}\text { The case-specific maternal near miss incidence ratios (ratio of near miss due to } \\
\text { hypertensive disorders per 1,000 live births) }\end{array}$} & \multirow[t]{4}{*}{8.60} & Severe preeclampsia 3.06 \\
\hline & & Eclampsia 5.11 \\
\hline & & Chronic hypertension 0.15 \\
\hline & & HELLP syndrome 0.27 \\
\hline \multirow{4}{*}{$\begin{array}{l}\text { The case-specific severe maternal outcome ratio(ratio of maternal death plus near } \\
\text { misses from hypertensive disorders per 1,000 live births) }\end{array}$} & \multirow[t]{4}{*}{9.37} & Severe preeclampsia 3.25 \\
\hline & & Eclampsia 5.61 \\
\hline & & Chronic hypertension 0.15 \\
\hline & & HELLP syndrome 0.35 \\
\hline \multirow[t]{4}{*}{ The case-specific maternal mortality ratio(maternal deaths per 100,000 live births) } & \multirow[t]{4}{*}{780} & Severe preeclampsia 1940 \\
\hline & & Eclampsia 503 \\
\hline & & Chronic hypertension 0.0 \\
\hline & & HELLP syndrome 770 \\
\hline \multirow{3}{*}{$\begin{array}{l}\text { The case-specific severe maternal mortality index (maternal deaths divided by total } \\
\text { deaths plus maternal near misses) as a percentage }\end{array}$} & \multirow[t]{3}{*}{8.3} & Severe preeclampsia 6.0 \\
\hline & & Eclampsia 9.0 \\
\hline & & HELLP syndrome 22.2 \\
\hline \multirow{3}{*}{$\begin{array}{l}\text { The case-fatality rate for hypertensive disorders(proportion of deaths out of the total } \\
\text { number of patients presenting with specific complications) as a percentage. }\end{array}$} & \multirow[t]{3}{*}{5.1} & Severe preeclampsia 2.3 \\
\hline & & Eclampsia 17.8 \\
\hline & & HELLP syndrome28.6 \\
\hline
\end{tabular}

accessing vital services in peripheral units, delay in accessing prompt care, and poor quality of obstetric care in general [21-25]. In addition, the health services are constrained by limited supplies and equipment, poor skills and few numbers of lower-level healthcare providers to recognize and manage complications prior to referral, and high patient loads [21-25].

This high burden of hypertensive disorders in pregnancy is similar to that in other Eastern African countries [26-29] where hypertensive disorders are among the commonest causes of severe morbidity and even mortality. This calls for increased investments to increase research into how to address this burden in particular and on how to improve the quality of obstetric care in general, particularly improving clinical, laboratory and critical care facilities. This is necessary as diagnosis of maternal near miss using the organ/system dysfunction criteria requires efficient diagnostic and laboratory facilities. Yet, even the routine assessment of hepatic, renal and hematological function may not be available in many resource-limited settings where cases of severe preeclampsia/eclampsia and other obstetric disorders are managed [30]. In consideration of this difficulty in routinely conducting some of the vital biochemical laboratory investigations, the WHO criteria for definition of a near miss (in limited-resource settings such as in Uganda) may need to be revised to focus mainly on clinical and management-based criteria.

There are several explanations for the high burden of morbidity in our study. Many patients presented with multisystem involvement, as commonly occurs in severe hypertensive disorders [31-33], often leading to multiple organ failure and death. There was a high caesarean section rate in our study. The reason for the high caesarean section rate is partly due to the high incidence of eclampsia, presence of obstetric co-morbidities such as previous caesarean section (which rule out possibility of induction of labor or vaginal birth). In addition, many

Table 3 Incidence of the hypertensive disorders of pregnancy in relation to maternal outcomes

\begin{tabular}{|c|c|c|c|c|}
\hline \multirow{3}{*}{$\begin{array}{l}\text { Hypertensive } \\
\text { disorder }\end{array}$} & All Hypertensive disorders & Maternal deaths & Maternal near miss & NLTC \\
\hline & Number (percentage) & Number (percentage) & Number (percentage) & Number (percentage) \\
\hline & $N=403$ & $N=20$ & $N=222$ & $N=161$ \\
\hline Severe Preeclampsia & $218(54.1)$ & $5(25.0)$ & $79(11.4)$ & $134(83.20)$ \\
\hline Eclampsia & $172(42.7)$ & $13(65.0)$ & $132(19.0)$ & $27(16.8)$ \\
\hline Chronic Hypertension & $4(1.0)$ & $0(0.0)$ & $4(0.6)$ & $0(0.0)$ \\
\hline HELLP Syndrome & $9(2.2)$ & $2(10.0)$ & $7(1.0)$ & $0(0.0)$ \\
\hline
\end{tabular}


Table 4 Diagnostic criteria used for the definition of severe maternal outcomes (maternal near miss cases or maternal deaths) in hypertensive disorders of pregnancy

\begin{tabular}{|c|c|c|c|c|c|c|}
\hline Characteristics & All participants & Severe preeclampsia & Eclampsia & Chronic hypertension & HELLP syndrome & $p$-value \\
\hline Cyanosis & & & & & & $<0.001$ \\
\hline Present & 169 (41.9) & $50(22.9)$ & $112(65.1)$ & $2(50.0)$ & $5(55.5)$ & \\
\hline Absent & $234(58.1)$ & $168(77.1)$ & $60(34.9)$ & $2(50.0)$ & $4(44.4)$ & \\
\hline$\infty$ Abnormal respiration & & & & & & $<0.001$ \\
\hline Yes & $186(46.2)$ & $61(28.0)$ & $115(66.9)$ & $3(75.0)$ & $7(77.7)$ & \\
\hline No & $217(53.8)$ & $157(72.0)$ & $57(33.1)$ & $1(25.0)$ & $2(22.2$ & \\
\hline Oliguria & & & & & & $<0.001$ \\
\hline Yes & $234(58.1)$ & $168(77.1)$ & $63(36.6)$ & $1(25.0)$ & $2(22.2)$ & \\
\hline No & $169(41.9)$ & $50(22.9)$ & $109(63.4)$ & $3(75.0)$ & $7(77.7)$ & \\
\hline Loss of consciousness & & & & & & $<0.001$ \\
\hline Yes & $110(27.3)$ & $31(14.2)$ & $75(55.2)$ & $0(0.0)$ & $4(44.4)$ & \\
\hline No & $293(72.7)$ & $187(85.8)$ & $97(44.8)$ & $4(100.0)$ & $5(55.5)$ & \\
\hline Shock & & & & & & $<0.001$ \\
\hline Yes & $170(42.2)$ & $54(24.7)$ & $105(61.0)$ & $3(75.0)$ & $8(88.8)$ & \\
\hline No & $233(57.8)$ & $162(75.3)$ & $67(39.0)$ & $1(25.0)$ & $1(11.1)$ & \\
\hline Coagulopathy & & & & & & $<0.001$ \\
\hline Yes & $136(33.7)$ & $36(16.5)$ & $91(52.9)$ & $2(50.0)$ & $7(77.7)$ & \\
\hline No & $267(66.3)$ & $182(83.5)$ & $81(47.1)$ & $2(50.0)$ & $2(22.2$ & \\
\hline SOxygen & & & & & & $<0.001$ \\
\hline Yes & $182(45.2)$ & $61(28.0)$ & $111(64.5)$ & $2(50.0)$ & $9(100.0)$ & \\
\hline No & $221(54.8)$ & $157(72.0)$ & $61(35.5)$ & $2(50.0)$ & $0(0.0)$ & \\
\hline Thrombocytopenia & & & & & & $<0.001$ \\
\hline Yes & $183(45.4)$ & $54(24.7)$ & $118(68.6)$ & $2(50.0)$ & $7(77.7)$ & \\
\hline No & $220(54.6)$ & $162(75.3)$ & $54(31.4)$ & $2(50.0)$ & $2(22.2)$ & \\
\hline Elevated Serum Creatinine & & & & & & $<0.001$ \\
\hline Yes & $152(37.7)$ & $46(21.1)$ & $98(57.0)$ & $2(50.0)$ & $6(66.7)$ & \\
\hline No & $251(62.3)$ & $172(78.9)$ & $74(43.0)$ & $2(50.0)$ & $3(33.3)$ & \\
\hline Elevated serum Bilirubin & & & & & & $<0.001$ \\
\hline Yes & $141(35.0)$ & 39 (17.9) & $93(54.1)$ & $2(50.0)$ & $7(77.7)$ & \\
\hline No & $262(65.0)$ & $179(82.1)$ & 79 (45.9) & $2(50.0)$ & $2(22.2$ & \\
\hline Elevated serum Lactate & & & & & & $<0.001$ \\
\hline Yes & $136(33.7)$ & $34(15.6)$ & $95(55.2)$ & $2(50.0)$ & $5(55.5)$ & \\
\hline No & $267(66.3)$ & $184(84.4)$ & $77(44.8)$ & $2(50.0)$ & $4(44.4)$ & \\
\hline Use of Dialysis & & & & & & $<0.001$ \\
\hline Yes & $33(8.2)$ & $7(3.3)$ & $23(13.4)$ & $1(25.0)$ & $2(22.2)$ & \\
\hline No & $370(91.8)$ & $211(96.7)$ & $149(86.6)$ & $3(75.0)$ & $7(77.7)$ & \\
\hline Need for ICU or HDU admission & & & & & & $<0.001$ \\
\hline Yes & $208(51.6)$ & $65(29.8)$ & $132(76.7)$ & $3(75.0)$ & $8(88.8)$ & \\
\hline No & $195(48.4)$ & $153(70.2)$ & $40(23.3)$ & $1(25.0)$ & $1(11.1)$ & \\
\hline
\end{tabular}

$\infty$ Breathing rate more than 40 or less than 6 per minute; $\Omega$ Oxygen saturation less than $90 \%$ for more than 60 min; ICU Intensive care unit, HDU High dependency obstetric unit 
patients presented with severe preeclampsia remote from term, in which case caesarean section is the preferred mode of delivery. For instance, the incidence of caesarean section was $13 \%$ higher for severe preeclampsia compared to eclampsia, due to more cases of prior caesarean deliveries in patients with severe preeclampsia. In addition, cases of severe preeclampsia had nearly half as many severe maternal outcomes (death or near miss) or maternal death (compared eclampsia), which indicates the high burden of co-morbidities in patients with hypertensive disorders.

Conceptua1lly, maternal near misses represent a point on the continuum where good health and death are the extreme points [31], where the complications could be described as uncomplicated, complicated (not lifethreatening), severely complicated (life-threatening) or fatal [31-35], and where outcomes depend on both the severity of illness and the quality of care [35]. Our findings in Table 4 show that many patients with severe preeclampsia and eclampsia presented with organ/system dysfunction related to the hepatic, renal, neurological, pulmonary and coagulatory system dysfunction. In addition, patients with eclampsia were more likely to present with organ-system dysfunction compared to those with severe preeclampsia. The WHO criteria were validated by the WHO Working Group using markers of dysfunction and total maximum SOFA (sequential organ failure assessment) in obstetric patients [36, 37]. From our study, presence of cyanosis, abnormal respiration, oliguria, loss of consciousness, circulatory collapse or shock, coagulopathy, thrombocytopenia, elevated serum lactate, the need for dialysis, and admission to the high dependency unit were significantly may be interpreted as potential risk markers for severity of hypertensive disorders, as they occurred more commonly in cases of eclampsia [38-42]. They are also markers of or prognostic factors for organ dysfunction and organ failure [43-46]. Several studies have shown that the higher the SOFA score, the higher the mortality [43-46]. Future studies should validate the SOFA scoring system among patients with severe obstetric morbidity in our setting. In addition, studies should evaluate and validate risk prediction models for adverse outcomes and prognostic factors using morbidity indices and SOFA scores.

\section{Conclusion}

Our study shows that hypertensive disorders cause a high burden of morbidity as indicated by the morbidity indices. This calls for interventions to reduce the burden and improve quality of care in hypertensive disorders of pregnancy. The findings highlight the feasibility of implementing a surveillance system for severe maternal morbidity from which data to inform interventions can be generated.

\section{Abbreviations}

ACOG, American College of Obstetrics and Gynecology; AKI, Acute kidney injury; ARDS, Acute respiratory distress syndrome; CKD, Chronic kidney disease; HDU, High dependency obstetric unit; HELLP syndrome, Syndrome of hemolysis, elevated liver enzymes and low platelets; ICU, intensive care unit; REC, Research and Ethics Committee; SD, standard deviation; SOFA, sequential organ failure assessment; WHO, World Health Organization.

\section{Acknowledgements}

This study was part of a post-doctoral research project funded by the Swedish International Development Agency (SIDA) through the Makerere UniversityKarolinska Institutet postdoctoral-research grants. The conclusions are those of the authors and do not necessarily represent the views of the funders or of Makerere University. We are grateful to all women who participated in this study

\section{Funding}

Funding for this research was provided by SIDA through Makerere University Karolinska Universitetet Research Collaboration post-doctoral research gents to Makerere University.

\section{Availability of data and materials}

Data from is available on request from Makerere University research repository, through the authors.

\section{Authors' contributions}

DKK conceptualized the study as part of his post-doctoral research project. $\mathrm{OK}, \mathrm{MOO}$, and NK advised on the design. DKK collected the data, led the analysis, and wrote the text of the paper. All the co-authors gave advice on the data analysis, presentation of the results, reviewed and edited the text and approved the final manuscript.

\section{Competing interests}

The authors declare that they have no competing interests.

\section{Consent for publication}

No individual identifier information is included in the manuscript. So consent for publication is not applicable.

\section{Ethics approval and consent to participate}

Ethical approval to conduct the study was obtained from the ethics and research committees of Mulago hospital (REC 310-2012), the School of Medicine, Makerere University College of Health Sciences (REC 2012-172) and from Uganda National Council for Science and Technology. Permission to conduct the study was obtained from the Department of Obstetrics and Gynaecology, Makerere University, and from Mulago National Referral Hospital and Jinja Hospital. Participants gave written informed consent to be enrolled in the study and for their data to be included in the study.

\section{Author details}

'Department of Obstetrics and Gynecology, School of Medicine, College of Health Sciences, Makerere University, P.O. Box 7072, Kampala, Uganda. ${ }^{2}$ Department of Obstetrics and Gynecology, Jinja Regional Hospital, Jinja, Uganda. ${ }^{3}$ Department of Nursing, School of Health Sciences, College of Health Sciences, Makerere University, P.O. Box 7072, Kampala, Uganda.

${ }^{4}$ Clinical, Operations and Health Services Research Program, Joint Clinical Research Centre, P. O. Box 10005, Kampala, Uganda.

Received: 4 December 2015 Accepted: 30 July 2016

Published online: 04 August 2016

\section{References}

1. American College of Obstetricians and Gynecologists, Task Force on Hypertension in Pregnancy. Hypertension in pregnancy. Report of the American College of Obstetricians and Gynecologists' Task Force on Hypertension in Pregnancy. Obstet Gynecol. 2013;122(5):1122-31.

2. Duley $L$. The global impact of pre-eclampsia and eclampsia. Semin Perinatol. 2009;33(3):130-7.

3. Khan KS, Wojdyla D, Say L, Gülmezoglu AM, Van Look PF. WHO analysis of causes of maternal death: a systematic review. Lancet. 2006;367(9516):1066-74. 
4. Fong A, Chau CT, Pan D, Ogunyemi DA. Clinical morbidities, trends, and demographics of eclampsia: a population-based study. Am J Obstet Gynecol. 2013;209(3):229. e1-7.

5. Abalos E, Cuesta C, Grosso A, Chou D, Say L. Global and regional estimates of preeclampsia and eclampsia: a systematic review. Eur J Obstet Gynecol. 2013;170(1):1-7.

6. Souza JP, Cecatti JG, Parpinelli MA, Sousa MH, Lago TG, et al. Maternal morbidity and near miss in the community: findings from the 2006 Brazilian demographic health survey. BJOG. 2010;117(13):1586-92.

7. Kaye DK, Kakaire O, Osinde MO. Systematic review of the magnitude and case fatality rate for severe maternal morbidity in sub-Saharan Africa between 1995 and 2010. BMC Pregnancy Childbirth. 2011;11:65.

8. Ronsmans C, Campbell O. Quantifying the fall in mortality associated with interventions related to hypertensive diseases of pregnancy. BMC Public Health. 2011:11 Suppl 3:S8.

9. Kullberg G, Lindeberg S, Hanson U. Eclampsia in Sweden. Hypertens Pregnancy. 2002;21(1):13-21.

10. Khosla AH, Dahiya K, Sangwan K. Maternal mortality and 'near-miss' in rura north India. Int J Gynaecol Obstet. 2000;68(2):163-4.

11. Eastabrook G, Brown M, Sargent I. The origins and end-organ consequence of pre-eclampsia. Best Pract Res Clin Obstet Gynaecol. 2011;25(4):435-47.

12. Bhattacharya S, Ayansina D, Black C, Hall S, Afolabi E, Millar C. Are women with gestational hypertension or preeclampsia at an increased long term risk of kidney function impairment? Hypertens Pregnancy. 2012;2(3):262.

13. Kuklina EV, Ayala C, Callaghan WM. Hypertensive disorders and severe obstetric morbidity in the United States. Obstet Gynecol. 2009;113:1299-306.

14. Zang J, Meikle S, Trumble A. Severe maternal morbidity associated with hypertensive disorders in pregnancy in the United States. Hypertens Pregnancy. 2003;22:203-12.

15. Say L, Souza JP, Pattinson RC. Maternal near miss - towards a standard tool for monitoring quality of maternal health care. Best Pract Res Clin Obstet Gynaecol. 2009;23:287-96.

16. Bernard GR, Artigas A, Brigham KL, Carlet J, Falke K, Hudson L, et al. The American-European Consensus Conference on ARDS. Definitions, mechanisms, relevant outcomes, and clinical trial coordination. Am Respir Crit Care Med. 1994;149(3 Pt 1):818-24.

17. Ahmed R, Dunford J, Mehran R, Robson S, Kunadian V. Pre-eclampsia and future cardiovascular risk among women: a review. J Am Coll Cardiol. 2014;63(18):1815-22.

18. Garovic VD, Hayman SR. Hypertension in pregnancy: an emerging risk factor for cardiovascular disease. Nat Clin Pract Nephrol. 2007;3(11):613-22.

19. Amaral LM, Cunningham Jr MW, Cornelius DC, LaMarca B. Preeclampsia: long-term consequences for vascular health. Vasc Health Risk Manag. 2015;11:403-15.

20. Brown MC, Best KE, Pearce MS, Waugh J, Robson SC, Bell R. Cardiovascular disease risk in women with pre-eclampsia: a systematic review and metaanalysis. Eur J Epidemiol. 2013;28(1):1-19.

21. Nakimuli A, Nakubulwa S, Kakaire O, Osinde MO, Mbalinda SN, Nabirye RC, et al. Maternal near misses from two referral hospitals in Uganda: a prospective cohort study on incidence, determinants and prognostic factors. BMC Pregnancy Childbirth. 2016;16(1):24.

22. Kaye DK, Nakimuli A, Kakaire O, Osinde MO, Mbalinda SN, Kakande N. Gaps in continuity of care: patients' perceptions of the quality of care during labor ward handover in Mulago hospital, Uganda. BMC Health Serv Res. 2015;15:190.

23. Nakimuli A, Mbalinda SN, Nabirye RC, Kakaire O, Nakubulwa S, Osinde MO, et al. Still births, neonatal deaths and neonatal near miss cases attributable to severe obstetric complications: a prospective cohort study in two referral hospitals in Uganda. BMC Pediatr. 2015:15:44.

24. Kigenyi O, Tefera GB, Nabiwemba E, Orach CG. Quality of intrapartum care at Mulago national referral hospital, Uganda: clients' perspective. BMC Pregnancy Childbirth. 2013;13:162.

25. Weeks A, Lavender T, Nazziwa E, Mirembe F. Personal accounts of 'nearmiss' maternal mortalities in Kampala, Uganda. BJOG. 2005;112(9):1302-7.

26. Ali AA, Khojali A, Okud A, Adam GK, Adam I. Maternal near-miss in a rural hospital in Sudan. BMC Pregnancy Childbirth. 2011;11:48.

27. David E, Machungo F, Zanconato G, Cavaliere E, Fiosse S, Sululu C, et al. Maternal near miss and maternal deaths in Mozambique: a cross-sectional, region-wide study of 635 consecutive cases assisted in health facilities of Maputo province. BMC Pregnancy Childbirth. 2014;14:401.
28. Nelissen E, Mduma E, Broerse J, Ersdal H, Evjen-Olsen B, van Roosmalen J, et al. Applicability of the WHO maternal near miss criteria in a low-resource setting. PLoS One. 2013;8(4):e61248.

29. Gebrehiwot $Y$, Tewolde BT. Improving maternity care in Ethiopia through facility based review of maternal deaths and near misses. Int J Gynaecol Obstet. 2014;127 Suppl 1:S29-34

30. World Health Organization. Evaluating the quality of care for severe pregnancy complications. The WHO near-miss approach for maternal health. 2011. http://www.who.int/reproductivehealth/publications/ monitoring/9789241502221/en.

31. Pollock WE. Caring for pregnant and postnatal women in intensive care: what do we know? Aust Crit Care. 2006;19(2):54-5. 57-65.

32. Pérez A, Bacallao J, Alcina S, Gómez Y. Severe maternal morbidity in the intensive care unit of a Havana Teaching Hospital, 1998 to 2004. MEDICC Rev. 2008;10(3):17-23.

33. Zanette E, Parpinelli MA, Surita FG, Costa ML, Haddad SM, Sousa MH, et al Maternal near miss and death among women with severe hypertensive disorders: a Brazilian multicenter surveillance study. Reprod Health. 2014;11(1):4

34. Geller SE, Rosenberg D, Cox SM, Brown ML, Simonson L, Driscoll CA, et al. The continuum of maternal morbidity and mortality: factors associated with severity. Am J Obstet Gynecol. 2004;191(3):939-44.

35. Souza JP, Cecatti JG, Parpinelli MA, Krupa F, Osis MJ. An emerging "maternal near-miss syndrome": narratives of women who almost died during pregnancy and childbirth. Birth. 2009;36(2):149-58.

36. Oliveira-Neto A, Parpinelli MA, Cecatti JG, Souza JP, Sousa MH. Sequential organ failure assessment score for evaluating organ failure and outcome of severe maternal morbidity in obstetric intensive care. Sci World J. 2012; 2012:172145.

37. Cecatti JG, Souza JP, Oliveira Neto AF, Parpinelli MA, Sousa MH, Say L, Pattinson RC. Pre-validation of the WHO organ dysfunction based criteria for identification of maternal near miss. Reprod Health. 2011:8:22.

38. Roberts JM, Taylor RN, Goldfien A. Clinical and biochemical evidence of endothelail cell dysfunction in the pregnancy syndrome pre-eclampsia. Am J Hypertens. 1991;4:700-7.

39. Bibi S, Memon A, Sheikh JM, Qureshi AH. Severe acute maternal morbidity and intensive care in a public sector university of Pakistan. J Ayub Med Coll Abbottabad. 2008:20(1):109-12.

40. American College of Chest Physicians/Society of Critical Care Medicine. Consensus Conference: Definitions for sepsis and organ failure and guidelines for the use of innovative therapies in sepsis. Crit Care Med. 1992;20(6):864-74.

41. Levy MM, Fink MP, Marshall JC, Abraham E, Angus D, Cook D, et al. SCCM/ ESICM/ACCP/ ATS/SIS International Sepsis Definitions Conference. Intensive Care Med. 2003:29(4):530-8.

42. Vincent JL, Moreno R, Takala J, Willatts S, De Mendoça A, Bruining H, et al The (SOFA) Sepsis- related Organ Failure Assessment score to describe organ dysfunction/failure. Intensive Care Med. 1996;22(7):707-10.

43. Blanco J, Muriel-Bombín A, Sagredo V, Taboada F, Gandío F, Tamayo L, et al. Incidence, organ dysfunction and mortality in severe sepsis: a Spanish multicenter study. Crit Care. 2009;12(6):R158.

44. Flaatten $\mathrm{H}$, Gjerde $\mathrm{S}$, Guttormsen AB, Haugen $\mathrm{O}$, Høivik $\mathrm{T}$, Onarheim $\mathrm{H}$, et al. Outcome after respiratory failure is more dependent on dysfunction in other vital organs than on the severity of the respiratory failure. Crit Care. 2003;7(4):R72

45. Cabré L, Mancebo J, Solsona JF, Saura P, Gich I, Blanch L, et al. Multicenter study of the multiple organ dysfunction syndrome in intensive care units: the usefulness of sequential organ failure assessment scores in decision making. Intensive Care Med. 2005;31(7):927-33.

46. Maccariello E, Rocha E, Valente C, Nogueira L, Rocha P, Bonomo H, et al. Effects of early changes in organ dysfunctions on the outcomes of critically ill patients in need of renal replacement therapy. Clinics (Sao Paulo). 2008; 63(3):343-50. 